by qPCR. Their activity was measured using a Rac1, RhoA or Cdc42 Pulldown \& G-Lisa Activation Assay Kit.

Results: 1) PGC-1 $\beta$ knockdown inhibited lamellipodia and filopodia formations of RA-FLS compared with Lv-sh-GFP transfection group (cells with lamellipodia: $24 \% \pm 7 \%$ vs. $40 \% \pm 5 \%, P=0.035$; cells with filopodia: $34 \% \pm 9 \%$ vs. $49 \% \pm 4 \%, P=0.041$ ), while PGC- $1 \beta$ over-expression promoted lamellipodia and filopodia formations of RA-FLS compared with LVGFP transfection group (cells with lamellipodia: $50 \% \pm 4 \%$ vs. $34 \% \pm$ $6 \%, P=0.040$; cells with filopodia: $67 \% \pm 7 \%$ vs. $52 \% \pm 6 \%, P=0.045$, Figure $1 \mathrm{~A})$.

2) PGC-1 $\beta$ knockdown or over-expression did not affect the mRNA expression of Rac1, RhoA or Cdc42 (all $P>0.05$, Figure 1B). However, PGC-1 $\beta$ knockdown suppressed the activity of Rac1, RhoA and Cdc42 (Pulldown assays: 65\% 78\% reduction; G-LISA assays: 28\% 53\% reduction, all $P<0.05)$, while $P G C-1 \beta$ over-expression significantly increased their activity (Pulldown assays: 1.55 1.72 folds; G-LISA assays: 1.43 1.68 folds, all $P<0.05$, Figure $1 C, D)$.

Conclusion: Our findings revealed that elevated PGC-1 $\beta$ in RA-FLS promoted pseudopodia formation by activating Rho family proteins which imply a novel target on regulating RA-FLS migration.

A

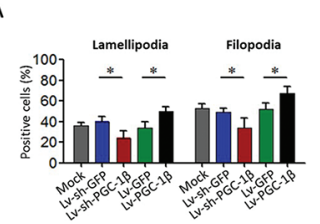

B

C
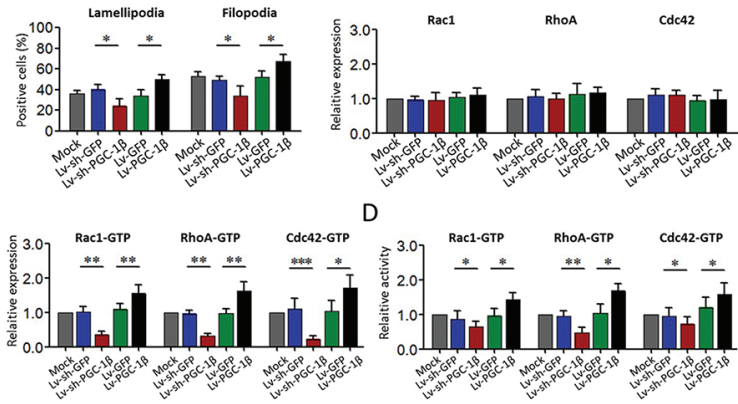

Figure 1 Effects of PGC-1 $\beta$ on pseudopodia formation and the activity of Rho GTPases in RA-FLS. RA-FLS were transfected with lentivirus for PGC-1 $\beta$ knockdown or over-expression. (A) Comparison of lamellipodia and filopodia formations by F-actin staining in RA-FLS. (B) The mRNA expression of Rac1, RhoA and Cdc42 was measured by qPCR. (C) The activity of Rac1, RhoA and Cdc 42 was measured by Pulldown assays. (D) The activity of Racl, RhoA and Cdc42 was measured by G- LISA assays. Data were representative as means $+\mathrm{SD}, * P<0.05, * * P<0.01, * * * P<0.001$

Acknowledgement: This work was supported by National Natural Science Foundation of China (81671612 and 81801606), Guangdong Natural Science Foundation (2017A030313576, 2017A030310236 and 2018A030313541) and Guangdong Medical Scientific Research Foundation (A2017109).

Disclosure of Interests: None declared

DOI: 10.1136/annrheumdis-2019-eular.3425

\section{SAT0041 GENOTYPE OF THE RHEUMATOID ARTHRITISSEVERITY LOCUS, RS26232, IS ASSOCIATED WITH INVASIVENESS OF RASFS IN VITRO}

Emma Dorris ${ }^{1}$, Eimear Flanagan ${ }^{1}$, Eimear Linnehan ${ }^{1}$, Michelle Trenkmann ${ }^{1}$, Karen Creevey ${ }^{1}$, Douglas Veale ${ }^{2}$, Ursula Fearon ${ }^{3}$, Gerry Wilson ${ }^{1} .{ }^{1}$ University College Dublin, Centre for Arthritis Research, Dublin, Ireland; ${ }^{2}$ University College Dublin, Rheumatology, Dublin, Ireland; ${ }^{3}$ Trinity College Dublin, Molecular Rheumatology, Dublin, Ireland

Background: The single nucleotide variant rs26232 has been associated with both the susceptibility to, and severity of, rheumatoid arthritis (RA). There is an allele dose response between rs26232 and radiological damage, with the minor, $\mathrm{T}$, allele being protective against disease severity. Rs26232 is situated in the first intron of C5orf30, which is a negative regulator of tissue damage and inflammation in RA.

Objectives: The objective of this study is to elucidate the mechanism by which rs26232 may mediate disease severity. We also aimed to determine the genotype-phenotype association of rs26232 in rheumatoid arthritis synovial fibroblasts (RASF).

Methods: RASF were derived from knee biopsies of RA patients taken at arthroscopy $(n=33)$. RASFs were used between passages 3-8. Matrigelcoated Boyden transwell chambers were used to measure invasion. Wound healing (scratch assays) were used to measure migration, whereas proliferation was measured via crystal violet staining of fixed RASF. Intracellular cytokine staining and cell surface markers were measured via flow cytometry. Secreted cytokines were measured in the supernatant of RASF using ELISA. Rs26232 genotype was determined by
PCR genotyping assay with allelic discrimination analysis. Anticitrullinated protein antibody (ACPA) status was measured for each participant. Quantitative real-time PCR was used to measure gene expression. Mann-Whitney $U$ test was used to compare two independent groups, Kruskal-Wallis test was used to compare groups of greater than two.

Results: $49 \%(n=16)$ of our RASF cohort where homozygous for the major allele CC, $42 \%(n=14)$ were heterozygous CT, 9\% $(n=3)$ were TT. Rs26232 is associated with invasion of RASFs with the CC genotype being more invasive than the CT genotype $(p=0.021)$. RASFs with the CC genotype had increased ICAM1 and VEGF cell surface expression compared to CT genotype ( $p=0.001, p=0.05$ respectively). MCP1/CCL2 was decreased in CC compared to CT $(p=0.013)$. No association was found between rs26232 genotype and migration, proliferation, or expression of MMP3, TIMP3, IP10 or MIP1a.

rs26232 is located in the first intron of C5orf30. There was no differential expression of total C5orf30 or with any of the 3 individual transcript variants in rs26232 genotype groups. In silico analysis of the region in which rs26232 is located on chromosome 5 identified a DNase Hypersensitivity cluster. Furthermore, mining of the Genotype-Tissue Expression (GTEX) expression quantitative trait loci (eQTL) database revealed a cluster of genes located upstream on chromosome 5 (102,850,000 $103,150,000)$ associated with rs26232. This region upstream from rs26232 contains 4 genes (EIF3KF1, PPIP5K2, PAM and GIN1), of which three are eQTLs of rs26232. In silico analysis revealed that the 3 eQTLs (EIF3KF1, PPiP5K2 and PAM) also contain the active enhancer mark H3K27Ac, whereas GIN1 does not.

Conclusion: The CC genotype of rs26232 is associated with both increased invasiveness of RASFs and increased adhesion markers compared to CT genotype. Rs26232 does not mediate its affect via its nearest gene, C5orf30. Rather, in silico analysis predicts rs26232 may function as a distal regulator of EIF3KF1, PPIP5K2 and PAM. Future work will test the hypothesis that rs26232 genotype phenotype association is mediated by EIF3KF1, PPIP5K2 and PAM.

Disclosure of Interests: None declared

DOI: 10.1136/annrheumdis-2019-eular.7514

\section{SAT0042 TWO POPULATIONS OF PD-1HICD4 T CELLS WITH DISTINCT B CELL HELPING CAPACITY, ARE ELEVATED IN THE PERIPHERAL BLOOD OF PATIENTS WITH EARLY RHEUMATOID ARTHRITIS}

Paula Fortea-Gordo ${ }^{1}$, Laura Nuño ${ }^{1}$, Alejandro Villalva ${ }^{1}$, Diana Peiteado ${ }^{1}$, Irene Monjo ${ }^{1}$, Amaya Puig-Kröger ${ }^{2}$, Paloma Sanchez-Mateos ${ }^{2}$, Alejandro Balsa ${ }^{1}$ Maria-Eugenia Miranda-Carus ${ }^{2} .{ }^{1}$ Hospital La Paz-IdiPAZ, Rheumatology, Madrid, Spain; ${ }^{2}$ Gregorio Marañón Hospital, Immuno-Oncology, Madrid, Spain

Background: A novel population of B helper cells, phenotypically CD4 + CXCR5-PD-1 $1^{\text {hi }}$, has been described in the synovial tissues and peripheral blood of seropositive RA patients with an established disease, and termed 'peripheral helper' (Tph) cells. Contrary to CD4+CXCR5+PD- ${ }^{\text {hi }}$ follicular helper $T$ (Tfh) cells, Tph cells are not located in lymphoid organs but accumulate in inflamed tissues. The frequency and kinetics of circu lating Tph cells have not been examined in patients with early, untreated RA.

Objectives: To study the frequency of circulating Tph (cTph) cells, and also of circulating Tfh cell counterparts (cTfh), in patients with early RA (eRA).

Methods: Peripheral blood was drawn from DMARD-naïve early RA patients (2010 ACR criteria) with a disease duration $<24$ weeks $(n=48)$ For each patient, an age and gender-matched healthy control was also studied $(\mathrm{HC}, \mathrm{n}=48)$. After isolation by Ficoll-Hypaque gradient, freshly isolated PBMCs were stained with antibodies to CD3, CD4, CXCR5, ICOS, PD-1 and CCR2, and examined by flow cytometry. Autologous cocultures of naïve or memory B cells were established with CXCR5 (+) or (-) memory CD4 T cells.

Results: Seropositive (RF+ and/or ACPA+, $n=31$ ) but not seronegative eRA patients $(n=17)$, demonstrated increased frequencies and absolute numbers of cTph and cTfh cells. cTph but not cTfh cells expressed CCR2. Those eRA patients who experienced a significant clinical improvement at 12 months, demonstrated a marked reduction of their cTph whereas their cTfh cell numbers remained unchanged; at the same time, rheumatoid factor titres decreased significantly but ACPA (anti-citrullinated peptide antibodies) titres did not vary. Both CXCR5+ and CXCR5-CD4+ $\mathrm{T}$ cells were able to induce maturation of memory $\mathrm{B}$ cells, whereas only CXCR5+CD4+ $T$ cells could differentiate naïve B cells.

Conclusion: Two populations of PD- $1{ }^{\text {hi }} \mathrm{CD} 4 \mathrm{~T}$ cells with distinct phenotype and $\mathrm{B}$ cell helping capacity, are increased in the peripheral blood of 